\title{
Velocity estimation using synthetic aperture imaging [blood flow]
}

\author{
Nikolov, Svetoslav; Jensen, Jørgen Arendt
}

Published in:

Proceedings of IEEE Ultrasonics Symposium

Link to article, DOI:

10.1109/ULTSYM.2001.991985

Publication date:

2001

Document Version

Publisher's PDF, also known as Version of record

Link back to DTU Orbit

Citation (APA):

Nikolov, S., \& Jensen, J. A. (2001). Velocity estimation using synthetic aperture imaging [blood flow]. In Proceedings of IEEE Ultrasonics Symposium (Vol. 2, pp. 1409-1412). IEEE.

https://doi.org/10.1109/ULTSYM.2001.991985

\section{General rights}

Copyright and moral rights for the publications made accessible in the public portal are retained by the authors and/or other copyright owners and it is a condition of accessing publications that users recognise and abide by the legal requirements associated with these rights.

- Users may download and print one copy of any publication from the public portal for the purpose of private study or research.

- You may not further distribute the material or use it for any profit-making activity or commercial gain

- You may freely distribute the URL identifying the publication in the public portal

If you believe that this document breaches copyright please contact us providing details, and we will remove access to the work immediately and investigate your claim. 


\title{
Velocity estimation using synthetic aperture imaging
}

\author{
Svetoslav Ivanov Nikolov* and Jørgen Arendt Jensen \\ Center for Fast Ultrasound Imaging, Ørsted•DTU, Build. 348, \\ Technical University of Denmark, DK-2800 Lyngby, Denmark
}

\begin{abstract}
In a previous paper we have demonstrated that the velocity can be estimated for a plug flow using recursive ultrasound imaging [1]. The approach involved the estimation of the velocity at every emission and using the estimates for motion compensation. An error in the estimates, however, would lead to an error in the compensation further increasing the error in the estimates.

In this paper the approach is further developed such that no motion compensation is necessary. In recursive ultrasound imaging a new high resolution image is created after every emission. The velocity was estimated by cross correlating RF lines from two successive emissions $n$ and $n+1$, and then average over a number of lines. In the new approach images $n$ and $n+N, n+1$ and $n+N+1$ are cross correlated, where $N$ is the number of emissions for one image. These images experience the same phase distortion due to motion and therefore have a high correlation without motion compensation. The advantage of the approach is that a color flow map can be created for all directions in the image simultaneously at every emission, which makes it possible to average over a large number of lines. This makes stationary echo canceling easier and significantly improves the velocity estimates. The approach is verified using simulations with the program Field II and measurements on a blood-mimicking phantom. The estimates from the simulations have a bias of $-3.5 \%$ and a mean standard deviation less than $2.0 \%$ for a parabolic velocity profile. The estimates from the measurements for the same setup exhibit a larger bias - $11 \%$, but the standard deviation is comparable to the simulations $(\sigma \sim 2.5 \%)$.
\end{abstract}

\section{Introduction}

Synthetic aperture ultrasound imaging (SAUI) is appealing with its capability to produce high-quality images, which are dynamically focused both in transmit, and in receive. The use of a synthetic transmit aperture imaging STAU has the additional advantage of low number of emissions and high frame rates. The SAUI in general, and the (STAU) in particu-

*E-mail: sn@oersted.dtu.dk lar, have therefore been investigated for many years, but have never left the area of research, and are not used in the medical scanners yet. One of the major hold-backs to the clinical use is that till present day there have not been any reports of estimation and visualization of blood flow. Without this feature a modern scanner cannot be considered full featured. This paper shows a way how to solve this problem by designing a new velocity estimator based on STAU.

The previous attempts for velocity estimation have been confined to the estimation of the gross tissue motion and used for motion compensation [2,3]. The approach suggested by Nock and Trahey [2] uses cross-correlation between the received raw RF signals. The algorithm, however, relies on the fact that the transmission is focused, and that the received signals come from the same direction. It is therefore not suitable for STAU. The method suggested by Bilge et al. [3] relies on the cross-correlation between low-resolution images, which are formed using the same transmit/receive pairs of transducer elements. The beam is, however, broad and the blood cells within its limits have a wide spread of velocities. This results in an increased bias and standard deviation of the estimates.

Both types of motion compensation schemes show improved performance, when the images are obtained using the same transmit/receive element pairs, or in other words have the same spatial frequencies. The high-resolution images (HRI) have the highest overlap of spatial frequencies, and therefore they should give the best estimates. The correlation of the signals received from the blood cells decreases rapidly due to migration of scatterers, beam-width modulation, and flow gradients (change in the relative positions between the scatterers) [4]. The HRIs must be generated after every emission, which is possible using recursive ultrasound imaging. These images suffer from motion artifacts, which change from frame to frame. In a previous article [1] we have shown that it is possible to compensate for the motion artifacts and to estimate the velocity from the motioncompensated HRIs. The success of the velocity estimation relies on the success of the motion compensation, which makes the whole approach unstable. In the next section it is shown how to modify a velocity estimator in such a way, that no motion compensation is needed prior to the velocity estimation. 


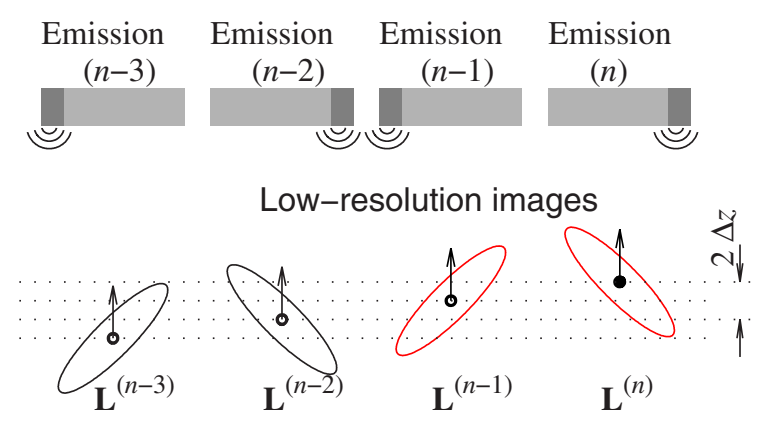

High-resolution images

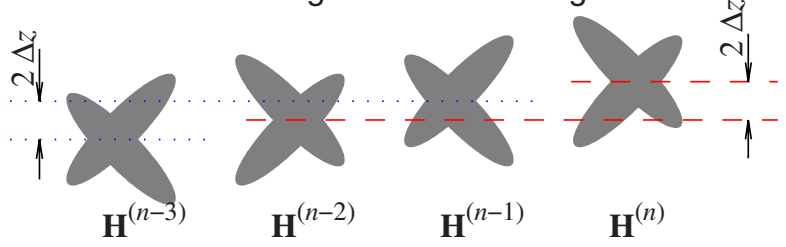

Figure 1: Creating several successive high-resolution images.

\section{Theory}

Fig. 1 shows the creation of several successive highresolution images, when a single scatterer moves towards the transducer. The top row illustrates the emission sequence. Only the outermost two elements are used in transmit $(N=2)$, and the active one is drawn with a dark gray color. They emit sequentially - first element number 1 then element number $N_{x d c}$, then again element number 1 and so on. $N_{x d c}$ is the total number of transducer elements. The emissions have a global index $n$, and are counted from the start of the process. The relation between the index of the transmit element and the emission number is given by:

$$
i=((n-1) \bmod N) N_{\text {step }}+1,
$$

where $N_{\text {step }}$ is the increment of the index of the transmitting elements. If every of the $N_{x d c}$ transducer elements was used, then $N_{\text {step }}$ would be 1 . All of the transducer elements are used upon reception, and after every emission $n$ a low-resolution image (LRI) $\mathbf{L}^{(n)}(t)$ is formed. The point spread functions for these LRIs are tilted at different angles to the transducer surface. The angles depend on the position of the transmitting element. The middle row of Fig. 1 illustrates the low resolution images of a single point. The point moves from emission to emission towards the transducer at a distance $\Delta z$. It can be shown that $\mathbf{L}^{(n)}(t)$ and $\mathbf{L}^{(n-N)}$ are translated versions of each other, when $\Delta z$ is on the order of a millimeter [5].

At every emission a high-resolution image can be formed

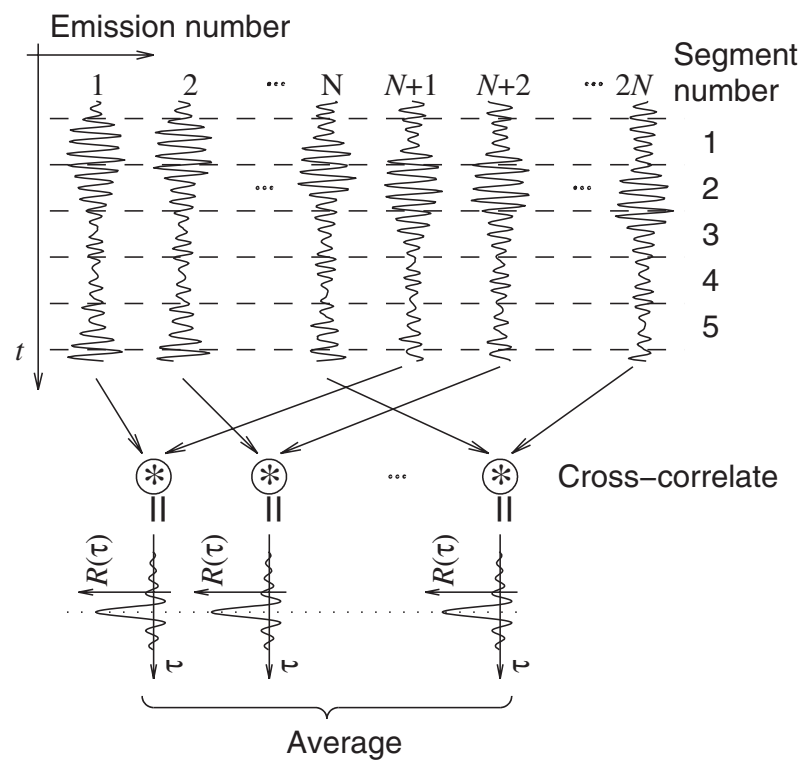

Figure 2: Estimation of the cross-correlation function of several consecutive high-resolution lines formed in the same direction.

by summing the last $N$ low-resolution images [6]:

$$
\mathbf{H}^{(n)}(t)=\sum_{i=n-N+1}^{n} \mathbf{L}^{(i)}(t),
$$

where $t$ is the time from the start of the emission. If the tissue is motionless, then $\mathbf{L}^{(i)}(t) \equiv \mathbf{L}^{(i-N)}(t)$ and $\mathbf{H}^{(n)}(t) \equiv$ $\mathbf{H}^{(n-N)}(t)$. In the presence of motion, however, $\mathbf{L}^{(n)}(t)=$ $\mathbf{L}^{(n-N)}(t-2(N \Delta z) / c)$, where $c$ is the speed of sound. It can be shown that if the velocity is constant, then:

$$
\mathbf{H}^{(n)}(t)=\mathbf{H}^{(n-N)}\left(t-2 \frac{N v_{z} T_{p r f}}{c}\right),
$$

where $T_{p r f}=1 / f_{p r f}$ is the pulse repetition period, and $v_{z}$ is the component of the velocity towards the transducer. This situation is depicted in the bottom row of Fig. 1. It can be seen that two successive high-resolution images differ due to the different transmit sequences with which they were obtained, but the high resolution images, that are $N$ transmissions apart, are shifted versions of one another. This shift can be estimated using cross-correlation of the high-resolution lines $H^{n}(t)$ and $H^{(n-N)}(t)$, which are formed in the same direction of the image (see Fig. 2).

The cross-correlation becomes [5]:

$$
\begin{aligned}
R_{n-N, n}(\tau) & =\frac{1}{2 T} \int_{T} H^{(n-N)}(t) H^{(n)}(t+\tau) d t \\
& =\frac{1}{2 T} \int_{T} H^{(n-N)}(t) H^{(n-N)}\left(t-t_{s}+\tau\right) d t \\
& =R_{n-N, n-N}\left(\tau-t_{s}\right),
\end{aligned}
$$


where $t_{s}=2 \frac{N v_{z} T_{p r f}}{c}$ is the time shift due to motion. The peak of the cross-correlation function is located at $\tau=t_{s}$. The velocity can be found from the time shift:

$$
v_{z}=\frac{c}{2} \frac{t_{s}}{N T_{p r f}} .
$$

The lines at emissions $n-i$ and $n-N-i$ are subject to the same time shift and the peak of their cross-correlation function is also located at a lag $\tau=t_{s}$, as shown in Fig. 2. The different cross-correlation functions can, thus, be averaged.

In the case of a flow profile, such as the parabolic flow profile, the velocity of the blood flow changes as a function of space and time. To estimate the velocity at the different spatial positions, each of the RF lines is divided into a number of segments as shown in Fig. 2. A cross-correlation function is estimated for each of the segments. Normally the signals are digital, and the discrete version of the cross-correlation is:

$$
\hat{R}_{n-N, n}\left[\eta, i_{\text {seg }}\right]=\frac{1}{N_{s}} \sum_{k=\left(i_{\text {seg }}-1\right) N_{s}}^{i_{\text {seg }} N_{s}-1} H^{(n-N)}[k] H^{(n)}[k+\eta],
$$

where $N_{s}$ is the number of samples in a segment and $i_{\text {seg }}$ is the number of the segment. If the the velocity does not change significantly for several sequential acquisitions $N_{c}$, then the estimate can be improved by averaging the estimated crosscorrelation functions:

$$
\hat{R}_{a v}^{(n)}\left[\eta, i_{s e g}\right]=\frac{1}{N_{c}} \sum_{l=-N_{c}+1}^{0} \hat{R}_{n-l-N, n-l}\left[\eta, i_{\text {seg }}\right] .
$$

Fig. 2 shows the case for which $N_{c}=N$. If the images were created after every $N$ emissions, then the total number of emissions needed for the velocity estimate would be $N_{c} N$. For $N=8$ and $N_{c}=16$, the total number of emissions would be $N_{c} N=128$. The correlation between the high-resolution lines would decrease due to acceleration, velocity gradients and migration of scatterers. In the new approach only $N+N_{c}=24$ emissions are necessary, thus preserving the high-correlation between the images and giving the possibility of estimating the flow with a low bias and variance.

Let the lag at which the maximum occurs be $\eta_{m}$. The estimated velocity is:

$$
\hat{v}_{z}=\frac{c}{2} \frac{\eta_{m} f_{p r f}}{N f_{s}}
$$

The estimate can be improved by fitting a second order curve to the estimate, and interpolating the time shift [4]:

$$
\eta_{s}=\eta_{m}-\frac{\hat{R}_{a v}^{(n)}\left[\eta_{m}+1\right]-\hat{R}_{a v}^{(n)}\left[\eta_{m}-1\right]}{2\left(\hat{R}_{a v}^{(n)}\left[\eta_{m}+1\right]-2 \hat{R}_{a v}^{(n)}\left[\eta_{m}\right]+\hat{R}_{a v}^{(n)}\left[\eta_{m}-1\right]\right)} .
$$

\begin{tabular}{lcr}
\hline \hline Parameter & Value & Unit \\
\hline Transducer center frequency & 7.5 & $\mathrm{MHz}$ \\
Relative transducer bandwidth & 66 & $\%$ \\
Number of elements & 64 & \\
Transducer pitch & 205 & $\mu \mathrm{m}$ \\
Transducer height & 4 & $\mathrm{~mm}$ \\
Excitation type & chirp & \\
Excitation start frequency & 0 & $\mathrm{~Hz}$ \\
Excitation end frequency & 17 & $\mathrm{MHz}$ \\
Excitation duration & 20 & $\mu \mathrm{s}$ \\
Sampling frequency & 100 & $\mathrm{MHz}$ \\
Pulse repetition frequency & 7000 & $\mathrm{~Hz}$ \\
Propagation velocity & 1540 & $\mathrm{~m} / \mathrm{s}$ \\
Maximum velocity & 0.18 & $\mathrm{~m} / \mathrm{s}$ \\
\hline \hline
\end{tabular}

Table 1: Relevant parameters for the simulation of the parabolic flow.

The stationary echo canceling is done only on the images that were obtained at every $N$ th emission, i.e. between images $n$ and $n-k N$, where $k \in \mathrm{Z}$.

One of the major problems of synthetic aperture imaging is the low signal-to-noise ratio. A linear frequency modulated pulse can be used in transmit in order to increase the transmitted energy, and hence, the signal-to-noise ratio.

\section{Results}

The method is verified through simulations and measurements. The simulation is done using the program Field II [7]. Some of the relevant simulation parameters are given in Table 1.

The simulated vessel has a diameter of $1 \mathrm{~cm}$, and length of $5 \mathrm{~cm}$. The center of the vessel is positioned $50 \mathrm{~mm}$ away from the transducer surface. The angle between the vessel walls and the transducer surface is $45^{\circ}$. The number of scatterers inside the vessel is 78,549. The positions of the scatterers are randomly selected with an uniform (white) distribution. Their amplitudes have a random Gaussian distribution with a zero mean.

Fig. 3 shows the result of the new estimator applied on the simulated data. The dash-and-dotted line corresponds to the true velocity. The thick line gives the mean of 200 estimates, and the dashed lines outline the mean of the estimates \pm three standard deviations $( \pm 3 \sigma)$. The maximum bias of the estimated profile for $\theta=45^{\circ}$ and $N_{c}=24$ is $-3.05 \%$ and the maximum standard deviation is $2.7 \%$. From Fig. 3 it can be seen that the performance for the case of $N_{c}=4$ is comparable: the maximum bias of the estimated profile is $-2.5 \%$. The standard deviation varies between $1.2 \%$ at 50 th $\mathrm{mm}$ and $5 \%$ at $42 \mathrm{nd} \mathrm{mm}$. 


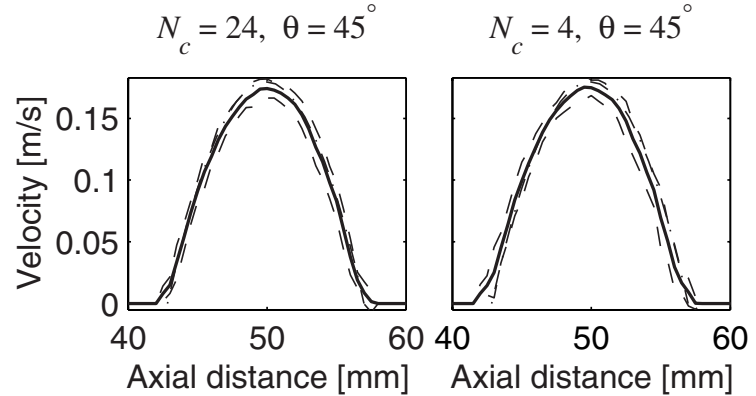

Figure 3: Velocity profiles obtained with the new crosscorrelation approach.

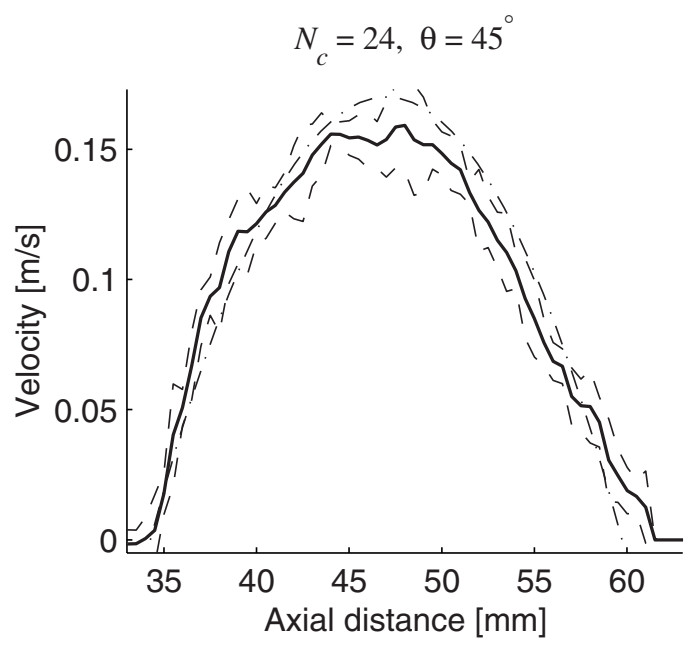

Figure 4: Velocity profile measured on a flow phantom.

Fig. 4 shows a velocity obtained by measuring the flow on a blood-flow mimicking phantom "EU-DFS-BMF-ver.1" by Danish Phantom Design (http://www.fantom.suite.dk). The fluid is driven by a Smedegaard Type EcoWatt 1 pump. The mean flow is measured by a Danfoss MAG 1100 flow meter. The length and diameter of the tube are $1 \mathrm{~m}$ and $17 \mathrm{~mm}$, respectively. Except for the propagation velocity of sound $c \approx 1485 \mathrm{~m} / \mathrm{s}$, and the sampling frequency $f_{s}=40 \mathrm{MHz}$, the parameters of the experiment are the same as in Table 1. The solid line in Fig. 4 is the mean estimated velocity, the dashed lines are \pm three standard deviations $(3 \sigma)$, and the dashed and dotted line is the true velocity profile. The standard deviation varies between 1.7 and $6 \%$. The bias at the peak (at depth of $47.5 \mathrm{~mm}$ ) is $-11 \%$.

\section{Conclusion}

In this paper we have presented an approach for synthetic aperture blood flow ultrasound imaging. Estimates with a low bias and standard deviation can be obtained with as few as 8 emissions $\left(N_{c}=4\right)$. The performance of the new estimator is verified using both simulations and measurements. The results demonstrate that a fully functioning synthetic aperture scanner can be made.

\section{Acknowledgment}

This work was supported by grant 9700883 and 9700563 from the Danish Science Foundation and by B-K Medical A/S, Gentofte, Denmark.

\section{References}

[1] S. I. Nikolov, K. Gammelmark, and J. A. Jensen. Velocity estimation using recursive ultrasound imaging and spatially encoded signals. In Proc. IEEE Ultrason. Symp., volume 2, pages 1473-1477, 2000.

[2] L. F. Nock and G. E. Trahey. Synthetic receive aperture imaging with phase correction for motion and for tissue inhomogenities - part II: effects of and correction for motion. IEEE Trans. Ultrason., Ferroelec., Freq. Contr., 39:496-501, 1992.

[3] H. Ş. Bilge, M. Karaman, and M. O'Donnell. Motion estimation using common spatial frequencies in synthetic aperture imaging. In Proc. IEEE Ultrason. Symp., pages 1551-1554, 1996.

[4] S. G. Foster, P. M. Embree, and W. D. O'Brien. Flow velocity profile via time-domain correlation: Error analysis and computer simulation. IEEE Trans. Ultrason., Ferroelec., Freq. Contr., 37:164-175, 1990.

[5] S. I. Nikolov. Synthetic aperture tissue and flow ultrasound imaging. $\mathrm{PhD}$ thesis, Ørsted•DTU, Technical University of Denmark, 2800, Lyngby, Denmark, 2001.

[6] S. I. Nikolov, K. Gammelmark, and J. A. Jensen. Recursive ultrasound imaging. In Proc. IEEE Ultrason. Symp., volume 2, pages 1621-1625, 1999.

[7] J. A. Jensen. Field: A program for simulating ultrasound systems. Med. Biol. Eng. Comp., 10th Nordic-Baltic Conference on Biomedical Imaging, Vol. 4, Supplement 1, Part 1:351-353, 1996b. 